\title{
Dicke-like effect in spin-polarized transport through coupled quantum dots
}

\author{
Piotr Trocha1, * and Józef Barnaś ${ }^{1,2, \text {, }}$ \\ ${ }^{1}$ Department of Physics, Adam Mickiewicz University, 61-614 Poznań, Poland \\ ${ }^{2}$ Institute of Molecular Physics, Polish Academy of Sciences, 60-179 Poznań, Poland
}

(Dated: August 23, 2021)

\begin{abstract}
Spin-dependent electronic transport through a quantum dot side-coupled to two quantum dots and attached to ferromagnetic leads with collinear (parallel and antiparallel) magnetizations is analyzed theoretically. The intra-dot Coulomb correlations are taken into account, whereas the inter-dot ones are neglected. Transport characteristics, i.e. conductance and tunnel magnetoresistance associated with the magnetization rotation from parallel to antiparallel configurations, are calculated by the noneqiulibrium Green function technique. The Green functions are derived by the equation of motion method in the Hartree-Fock approximation. The conductance spectra are shown to reveal features similar to the Dicke resonance in atomic physics.

PACS numbers: 73.21.La, 73.23.-b, 73.63.Kv, 85.35.Ds
\end{abstract}

\section{INTRODUCTION}

Interference effects in electronic transport through coupled quantum dots (QDs) are of great interest from both fundamental and application points of view. One of the simplest systems where the interference effects are clearly visible, consists of two QDs connected in parallel to electron reservoirs (metallic or semiconducting leads). This interferometer-like geometry allows observation of such quantum phenomena like the Aharonov-Bohm oscillations and the Fano antiresonance 1,2,3,4,5,6,7,8,9,10,11,12,13.

Conductance of systems consisting of three or more QDs reveals further interesting features of quantum transport $14,15,16,17,18,19,20,21,22,23,24$. Of particular importance seems to be the interference phenomenon $25,26,27,28,29$, which resembles the well known Dicke resonance in atomic physics $30,31,32$. The key feature of the Dicke resonance is the presence of a strong and very narrow emission line of a collection of atoms which are separated by a distance smaller than the wavelength of the emitted light 30,31 .

In this paper we consider the Dicke-like resonance in spin polarized transport through a system of three coupled QDs. One of the QDs is attached to two ferromagnetic leads, while the other two dots are side-coupled to the first one. Our considerations include the intra-dot electron correlation, while the inter-dot Coulomb interaction is assumed to be much smaller than the intra-dot one and is omitted. Transport characteristics, in particular conductance of the system and tunnel magnetoresistance associated with rotation of the electrodes' magnetizations from antiparallel to parallel alignment, are calculated by the non-equilibrium Green function technique in the linear response regime. Since the systems with Coulomb interactions cannot be solved exactly, we applied the Hartree-Fock (HF) approximation scheme to calculate the relevant Green functions from the equations of motion.

In section 2 we describe the model, while theoretical formulation of the problem and basic analytical formula are presented in section 3 . Numerical results for symmet- ric and asymmetric systems are shown and discussed in section 4. It is shown there that narrow Dicke-like peaks appear in the linear conductance. Final conclusions are given in section 5 .

\section{DESCRIPTION OF THE MODEL}

In this paper we consider a system consisting of three single-level quantum dots, coupled as shown schematically in figure 1. The dots QD1 and QD3 are coupled to the dot QD2 via direct hopping term. The dot QD2, in turn, is additionally attached to ferromagnetic leads (see figure 1). Generally, couplings between the dots as well as those between the dots and external leads can be controlled by applied gate voltages. We also note that technological difficulties associated with attaching a semiconductor quantum dot to ferromagnetic metallic leads have been overcome and first experimental results on spin polarized transport through individual semiconductor quantum dots have been published very recently ${ }^{?}$. Thus, experimental investigation of spin polarized transport in systems of coupled quantum dots seems to be only a matter of time. For simplicity we consider only collinear (parallel and antiparallel) magnetic orientations of the leads' magnetic moments. Apart from this, we include the intra-dot Coulomb interactions, while the inter-dot Coulomb repulsion is assumed to be weak and therefore is neglected. The system under consideration is then described by Hamiltonian of the general form $\hat{H}=\hat{H}_{\mathrm{L}}+\hat{H}_{\mathrm{R}}+\hat{H}_{\mathrm{QD}}+\hat{H}_{\mathrm{T}}$. The terms $\hat{H}_{\mathrm{L}}$ and $\hat{H}_{\mathrm{R}}$ describe respectively the left and right electrodes in the non-interacting quasi-particle approximation, $\hat{H}_{\alpha}=\sum_{\mathbf{k} \sigma} \varepsilon_{\mathbf{k} \alpha \sigma} c_{\mathbf{k} \alpha \sigma}^{\dagger} c_{\mathbf{k} \alpha \sigma}$ (for $\alpha=\mathrm{L}, \mathrm{R}$ ). Here, $c_{\mathbf{k} \alpha \sigma}^{\dagger}\left(c_{\mathbf{k} \alpha \sigma}\right)$ is the creation (annihilation) operator of an electron with the wave vector $\mathbf{k}$ and $\operatorname{spin} \sigma$ in the lead $\alpha$, whereas $\varepsilon_{\mathbf{k} \alpha \sigma}$ denotes the corresponding single-particle energy.

The third term of the Hamiltonian $H$ describes the 


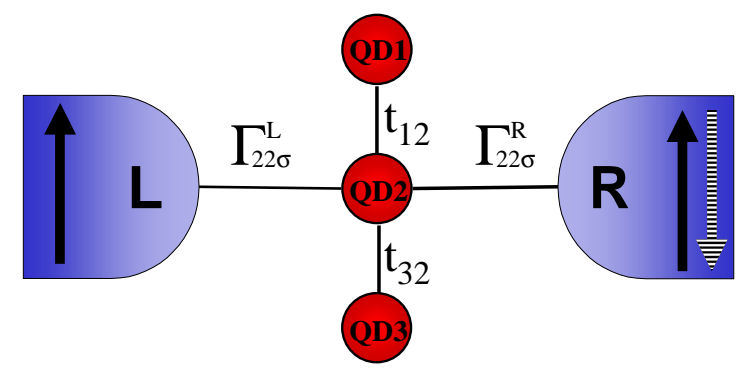

FIG. 1: Schematic picture of the quantum dot system coupled to ferromagnetic leads. $\Gamma_{22 \sigma}^{\alpha}(\alpha=L, R)$ describe spin dependent coupling of the dot QD2 to the ferromagnetic leads, whereas $t_{j 2 \sigma}$ is the hopping parameter between the $j$-th dot $(j=1,3)$ and the dot QD2.

system of three coupled quantum dots,

$$
\begin{aligned}
\hat{H}_{Q D} & =\sum_{i \sigma} \epsilon_{i \sigma} d_{i \sigma}^{\dagger} d_{i \sigma}+\sum_{i=1}^{3} U_{i} n_{i \sigma} n_{i \bar{\sigma}} \\
& -\sum_{j(=1,3)} \sum_{\sigma}\left(t_{j 2 \sigma} d_{2 \sigma}^{\dagger} d_{j \sigma}+\text { h.c. }\right)
\end{aligned}
$$

where $\epsilon_{i \sigma}$ is the energy level of the $i$-th $\operatorname{dot}(i=1,2,3)$, and $t_{j 2 \sigma}$ is the hopping parameter between the $j$-th dot $(j=1,3)$ and the dot QD2. Both $\epsilon_{i \sigma}$ and $t_{j 2 \sigma}$ are assumed to be spin dependent in a general case. The second term of the Hamiltonian $\hat{H}_{Q D}$ describes the intra-dot Coulomb interactions, with $U_{i}(i=1,2,3)$ denoting the corresponding Coulomb integrals.

The last term of the system's Hamiltonian, $H_{\mathrm{T}}$, describes electron tunneling from the leads to the dot QD2 (or vice versa), and takes the form

$$
\hat{H}_{\mathrm{T}}=\sum_{\mathbf{k} \alpha} \sum_{\sigma}\left(V_{2 \mathbf{k} \sigma}^{\alpha} c_{\mathbf{k} \alpha \sigma}^{\dagger} d_{2 \sigma}+\text { h.c. }\right)
$$

where $V_{2 \mathbf{k} \sigma}^{\alpha}$ are the relevant matrix elements. Coupling of the dot to external leads can be parameterized in terms of $\Gamma_{\sigma}^{\alpha}(\epsilon)=2 \pi \sum_{\mathbf{k}} V_{2 \mathbf{k} \sigma}^{\alpha} V_{2 \mathbf{k} \sigma}^{\alpha *} \delta\left(\epsilon-\epsilon_{\mathbf{k} \alpha \sigma}\right)$. We assume that $\Gamma_{\sigma}^{\alpha}(\epsilon)$ is constant within the electron band, $\Gamma_{\sigma}^{\alpha}(\epsilon)=\Gamma_{\sigma}^{\alpha}=$ const for $\epsilon \in\langle-D, D\rangle$, and $\Gamma_{\sigma}^{\alpha}(\epsilon)=0$ otherwise. Here, $2 D$ denotes the electron band width. Introducing the spin polarization $p_{r}$ of lead $r(r=L, R)$ as $p_{r}=\left(\rho_{r}^{+}-\right.$ $\left.\rho_{r}^{-}\right) /\left(\rho_{r}^{+}+\rho_{r}^{-}\right)$, the coupling parameters can be expressed as $\Gamma_{r}^{+(-)}=\Gamma_{r}\left(1 \pm p_{r}\right)$, with $\Gamma_{r}=\left(\Gamma_{r}^{+}+\Gamma_{r}^{-}\right) / 2$. Here, $\rho_{r}^{+}$ and $\rho_{r}^{-}$are the densities of states at the Fermi level for spin-majority and spin-minority electrons in the lead $r$, while $\Gamma_{r}^{+}$and $\Gamma_{r}^{-}$describe coupling of the dot QD2 to the lead $r$ in the spin-majority and spin-minority channels, respectively.

\section{ANALYTICAL DESCRIPTION}

Electric current $J$ flowing through the system is given by the formula 33,34

$$
\begin{array}{r}
J=\frac{i e}{2 \hbar} \int \frac{d \epsilon}{2 \pi} \operatorname{Tr}\left\{\left[\boldsymbol{\Gamma}^{L}(\epsilon)-\boldsymbol{\Gamma}^{R}(\epsilon)\right] \mathbf{G}^{<}(\epsilon)\right. \\
\left.+\left[f_{L}(\epsilon) \boldsymbol{\Gamma}^{L}(\epsilon)-f_{R}(\epsilon) \boldsymbol{\Gamma}^{R}(\epsilon)\right]\left[\mathbf{G}^{r}(\epsilon)-\mathbf{G}^{a}(\epsilon)\right]\right\},
\end{array}
$$

where, $f_{\alpha}(\epsilon)=\left[e^{\left(\epsilon-\mu_{\alpha}\right) / k_{B} T}+1\right]^{-1}$ is the Fermi-Dirac distribution function in the lead $\alpha, \mathbf{G}^{<}(\epsilon)$ and $\mathbf{G}^{r(a)}(\epsilon)$ are the Fourier transforms of the lesser and retarded (advanced) Green functions of the dots, and $\boldsymbol{\Gamma}^{\alpha}(\epsilon)$ (for $\alpha=L, R)$ is a matrix which describes coupling of the dots to the leads. In the case under consideration the matrix $\boldsymbol{\Gamma}^{\alpha}(\epsilon)$ takes a simple form with only one nonzero matrix element,

$$
\Gamma_{\sigma}^{\alpha}=\left(\begin{array}{ccc}
0 & 0 & 0 \\
0 & \Gamma_{22 \sigma}^{\alpha} & 0 \\
0 & 0 & 0
\end{array}\right)
$$

In the case under consideration one can write $\Gamma_{22 \sigma}^{L}=$ $\Gamma\left(1 \pm p_{L}\right)$ for coupling of the dot to the left lead, and $\Gamma_{22 \sigma}^{R}=\gamma \Gamma\left(1 \pm p_{R}\right)$ (parallel configuration) or $\Gamma_{22 \sigma}^{R}=$ $\gamma \Gamma\left(1 \mp p_{R}\right)$ (antiparallel configuration) for coupling to the right lead. Here, $\sigma=\uparrow$ (upper sign), $\sigma=\downarrow$ (lower sign), $\Gamma$ is a constant, whereas $\gamma$ describes asymmetry in the coupling of the dot to the left and right leads.

To calculate electric current one needs to know the Green functions $G_{i j \sigma}^{r(a)}(\epsilon)$. These can be found by the equation of motion method. First, we apply the equation of motion to the causal Green function $G_{i j \sigma}(\epsilon)$. This equation of motion generates higher order Green functions, for which we write the corresponding equations of motion. New higher order Green functions are then decoupled in terms of the Hartree-Fock decoupling scheme. This decoupling scheme, described in details in Refs 2,12 , allows to express the higher-order Green functions through the lower-order ones. This, in turn, allows closing the relevant system of equations for the Green function $G_{i j \sigma}(\epsilon)$ and to write it in the matrix form as

$$
\begin{gathered}
\left(\begin{array}{ccc}
\epsilon-\epsilon_{1 \sigma}-A_{1} & t_{12 \sigma} B_{1} & 0 \\
t_{12 \sigma} B_{2} & \epsilon-\epsilon_{2 \sigma}-\Sigma_{\sigma}-A_{2} & t_{32 \sigma} B_{2} \\
0 & t_{32 \sigma} B_{3} & \epsilon-\epsilon_{3 \sigma}-A_{3}
\end{array}\right) \\
\times\left(\begin{array}{l}
G_{1 j \sigma} \\
G_{2 j \sigma} \\
G_{3 j \sigma}
\end{array}\right)=\left(\begin{array}{l}
\delta_{1 j} B_{1} \\
\delta_{2 j} B_{2} \\
\delta_{3 j} B_{3}
\end{array}\right),
\end{gathered}
$$

where for simplicity the $\epsilon$-dependence of the Green functions and other parameters has not been indicated explicitly (it will be restored where necessary). Apart from this, we have introduced the following notation:

$$
A_{i}=\frac{U_{i}\left(n_{i 2 \bar{\sigma}}-n_{2 i \bar{\sigma}}\right)}{\epsilon-\epsilon_{i \sigma}-U_{i}}
$$


for $i=1,3$,

$$
\begin{aligned}
& A_{2}=U_{2}\left(\epsilon-\epsilon_{2 \sigma}-U_{2}\right)^{-1}\left[t_{12 \sigma}\left(n_{12 \bar{\sigma}}-n_{21 \bar{\sigma}}\right)\right. \\
& \left.+t_{32 \sigma}\left(n_{32 \bar{\sigma}}-n_{23 \bar{\sigma}}\right)+\left(C_{2 \bar{\sigma}}^{\dagger}-C_{2 \bar{\sigma}}\right) n_{2 \bar{\sigma}} \Sigma_{\sigma}\right],
\end{aligned}
$$

and

$$
B_{i}=1+\frac{U_{i} n_{2 \bar{\sigma}}}{\epsilon-\epsilon_{i \sigma}-U_{i}}
$$

for $i=1,2,3$. To simplify notation we put here $\left\langle n_{i j \sigma}\right\rangle=\left\langle d_{i}^{+} d_{j}\right\rangle=n_{i j \sigma},\left\langle n_{i \sigma}\right\rangle=\left\langle d_{i}^{+} d_{i}\right\rangle=n_{i \sigma}$, and $C_{2 \bar{\sigma}} \equiv \sum_{k \alpha} V_{2 k \bar{\sigma}}^{\alpha}\left\langle c_{k \alpha \bar{\sigma}}^{\dagger} d_{2 \bar{\sigma}}\right\rangle$. We have also defined the self-energy $\Sigma_{\sigma}$ as

$$
\Sigma_{\sigma}=\Sigma_{\sigma}^{L}+\Sigma_{\sigma}^{R}
$$

with

$$
\Sigma_{\sigma}^{\alpha}=\sum_{k} \frac{V_{2 k \sigma}^{\alpha} V_{2 k \sigma}^{\alpha *}}{\epsilon-\epsilon_{k \alpha \sigma}}
$$

for $\alpha=L, R$.

Having found the causal Green functions from equation 5, one can calculate the retarded (advanced) Green functions as $G_{i j \sigma}^{r(a)}(\epsilon)=G_{i j \sigma}\left(\epsilon \pm i 0^{+}\right)$. The corresponding self-energy $\Sigma_{\sigma}^{r(a)}(\epsilon)=\Sigma_{\sigma}\left(\epsilon \pm i 0^{+}\right)$takes then the form,

$$
\Sigma_{\sigma}^{r(a)}(\epsilon)=\Lambda_{22 \sigma}(\epsilon) \mp \frac{i}{2} \Gamma_{22 \sigma},
$$

with

$$
\Gamma_{22 \sigma}=\Gamma_{22 \sigma}^{L}+\Gamma_{22 \sigma}^{R}
$$

and

$$
\Lambda_{22 \sigma}(\epsilon)=-\frac{1}{2 \pi} \Gamma_{22 \sigma} \ln \left(\frac{D-\epsilon}{D+\epsilon}\right) .
$$

Now, we need to find the lesser Green function. This can be found from the corresponding equation of motion, with the higher order Green functions calculated on taking into account the Langreth theorem ${ }^{34}$ and the Hartree-Fock decoupling scheme used when calculating the causal Green function.

\section{NUMERICAL RESULTS}

Using the formulas derived above, we calculate numerically the basic transport characteristics, i.e. the conductance and tunnel magnetoresistance. The latter quantity describes the resistance change when magnetic configuration of the system varies from antiparallel to parallel, and is described quantitatively by the ratio $\left(R_{\mathrm{AP}}-R_{\mathrm{P}}\right) / R_{\mathrm{P}}$, where $R_{\mathrm{AP}}$ and $R_{\mathrm{P}}$ denote the resistance in the antiparallel and parallel magnetic configurations, respectively. We assume that the dot levels are spin degenerate, $\epsilon_{i \sigma}=\epsilon_{i}$ (for $i=1,2,3$ ), and introduce two parameters, $h_{1}$ and
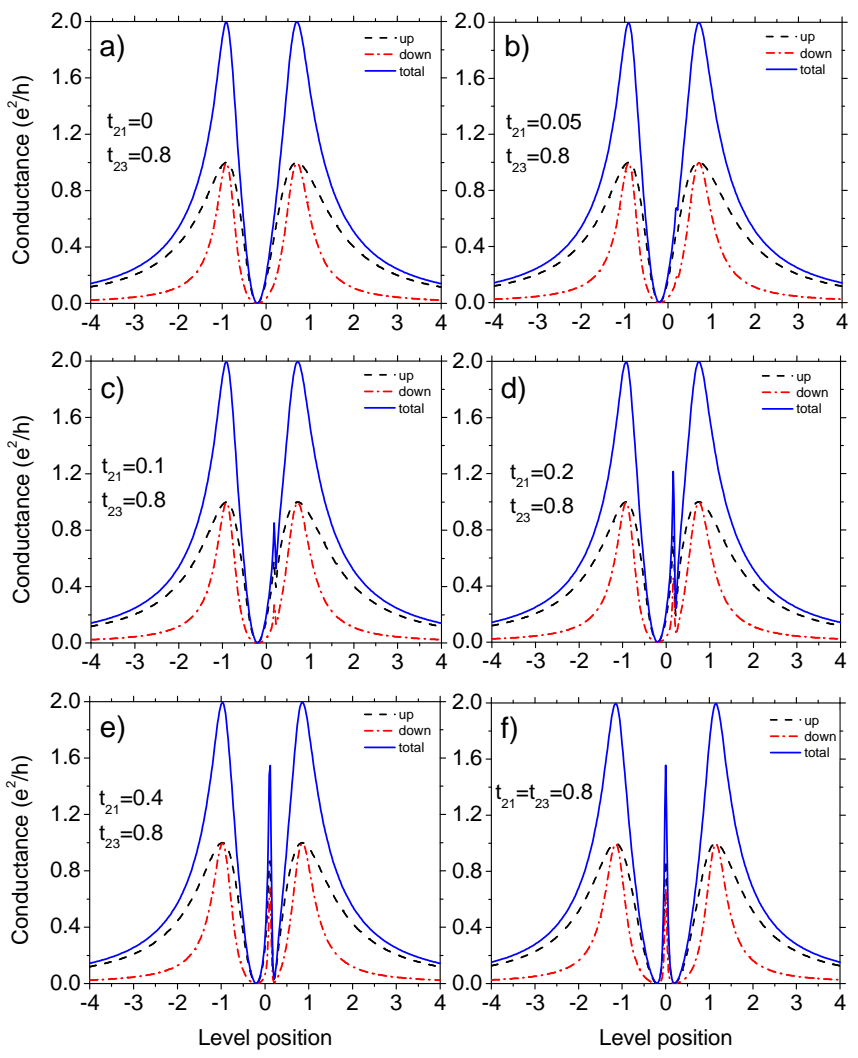

FIG. 2: Linear conductance in the parallel magnetic configuration, calculated as a function of the energy level $\epsilon_{2}$ of QD2 for indicated values of the intra-dot coupling parameters $t_{21}$ and $t_{23}$ and for $p=0.4, U_{1}=U_{2}=U_{3}=0, h_{1}=h_{2}=0.2$, $\gamma=1$, and $k_{B} T=0.01$. The energy is measured in the units of $\Gamma$.

$h_{2}$, which describe separation of the levels $\epsilon_{1}$ and $\epsilon_{3}$ from the level $\epsilon_{2}$, i.e. $\epsilon_{1}=\epsilon_{2}-h_{1}$ and $\epsilon_{3}=\epsilon_{2}+h_{2}$. Moreover, we assume $p_{L}=p_{R}=p$ and $\gamma=1$.

Consider first the limit of vanishing Coulomb interaction, $U_{i}=0$ for $i=1,2,3$. In figure 2 we show the linear conductance in the parallel magnetic configuration, calculated as a function of the energy level $\epsilon_{2}$ of QD2, measured from the Fermi level of the leads. The total conductance as well as that of individual spin channel are shown there. The energy level of the dot QD1 is shifted down by 0.2 with respect to $\epsilon_{2}$, while that of QD3 is shifted up by the same amount. Here and in the following the energy is measured in the units of $\Gamma\left(\Gamma=\Gamma_{L}=\Gamma_{R}\right)$. The hoping parameter $t_{23}$ is constant while the parameter $t_{21}$ increases from zero in figure $2(\mathrm{a})$ to $t_{21}=t_{23}$ in figure 2(f). Let us analyze now the main features of the conductance spectra. In figure $2(\mathrm{a})$ the dot QD1 is entirely detached from the dot QD2 and therefore has no influence on transport. The other two dots are coupled and form bonding and anti-bonding like states. Due to a nonzero value of $h_{2}$, these states are coupled differently to the leads. The linear conductance shows then two relatively broad peaks related to the bonding and anti- 
bonding like states, with a deep minimum in between, where the conductance turns to zero due to destructive interference of electron waves transmitted via bonding and anti-bonding states. We note, that the broadening of peaks is determined mainly by the coupling to the leads, but temperature also plays a role.

When the dot QD1 becomes weakly coupled to the dot QD2, a narrow peak emerges in the conductance at the position corresponding to the energy level of the dot QD1 (see figure 2(b-d)). The onset of this peak is a direct consequence of a nonzero value of $t_{21}$. The dots QD1 and QD3 are then both coupled to the dot QD2 and, disregarding coupling to the leads, one finds three new energy level of the whole three-dot system. The level corresponding to the new peak is only weakly coupled to the leads. When $t_{21}$ increases further, this peak moves towards zero energy, and in the strictly symmetrical case, $t_{21}=t_{23}$, the peak appears strictly at the position corresponding to the bare level of the dot QD2, $\epsilon_{2}=0$. The spectrum becomes then symmetrical, which is a consequence of the symmetry in the system. The central peak, however, remains much narrower than the other two peaks. Similar scenario also holds for constant $t_{21}$ and $t_{23}$ increasing from zero to $t_{23}=t_{21}$. The new peak emerges now on the left side of the minimum and moves towards $\epsilon_{2}=0$, as before. All the features discussed above appear not only in the total conductance, but also in the conductance of each spin channel separately, as clearly seen in figure 2 . The heights of the side peaks in figure 2 are equal to 2 conductance quanta $\left(e^{2} / h\right)$, independently of the values of the inter-dot hoping parameters. The height of central narrow peak, however, is smaller than that of the side broad peaks.

The central narrow peak resembles the Dicke resonance in atomic physics, where a strong narrow emission line appears when the distance between atoms is smaller than the Fermi wavelength of the corresponding radiation. In our case the role of distance between atoms is plied by the distance in energy levels, $h_{1}$ and $h_{2}$. When $h_{1}$ and $h_{2}$ increase, the width of the central line also increases. The two side peaks, in turn, become narrower and narrower. This is shown explicitly in figure 3, where the linear conductance for the symmetrical situation is presented for increasing $h_{1}$ and $h_{2}$ (the other parameters are the same as in figure 2). This behavior is quite reasonable, as the eigen-states of the dot system become more and more localized on the corresponding individual dots with increasing $h_{1}$ and $h_{2}$. Since the dots QD1 and QD3 are not coupled directly to the leads, the corresponding side peaks in the conductance become narrower and their width decreases with increasing $h_{1}$ and $h_{2}$. The central zero-energy level becomes then localized on the dot QD2, and the corresponding peak in the conductance becomes broader. Moreover, height of the central peaks increases and becomes equal to two conductance quanta, while that of the side peaks decreases with increasing $h_{1}$ and $h_{2}$.

When $h_{1}=h_{2} \neq 0$ and $t_{12}=t_{23}=t \neq 0$ (as in figure 3 ), the two side peaks in the conductance appear
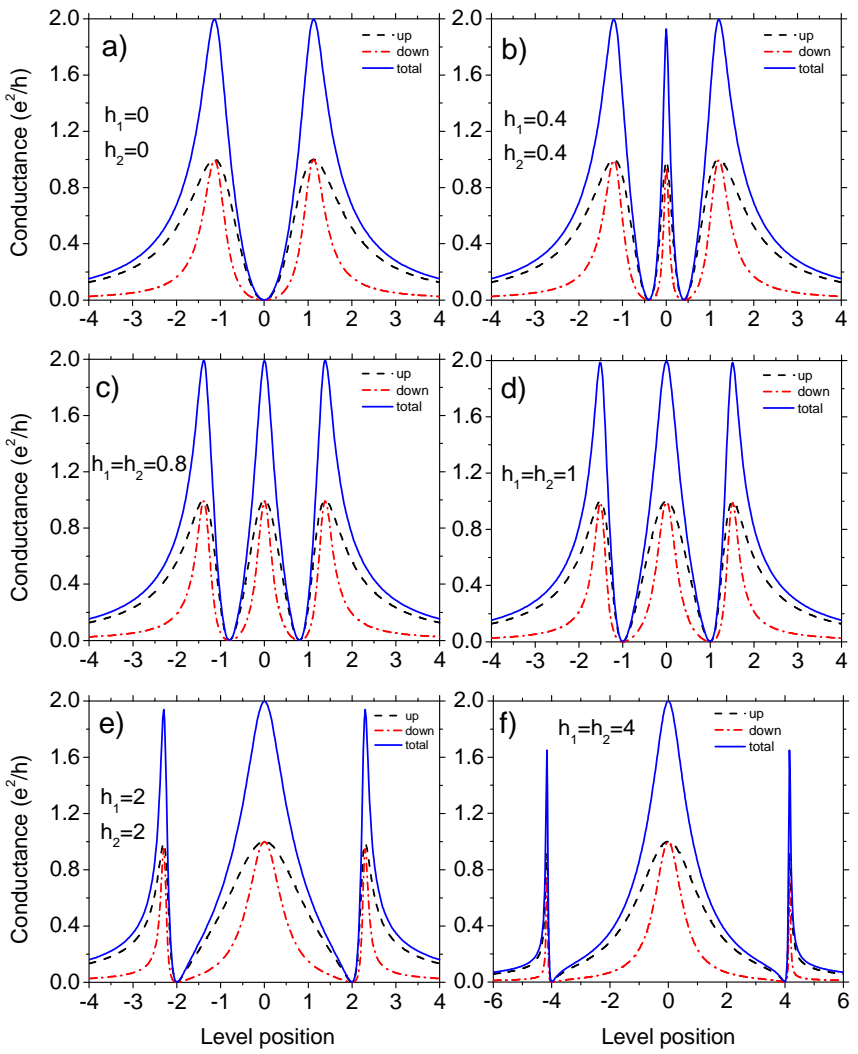

FIG. 3: Linear conductance in the parallel magnetic configuration, calculated as a function of the energy level $\epsilon_{2}$ of QD2 for indicated values of the level shifts $h_{1}$ and $h_{2}$, and for $t_{21}=t_{23}=0.8, p=0.4, \gamma=1, U_{1}=U_{2}=U_{3}=0$, and $k_{B} T=0.01$. The energy is measured in the units of $\Gamma$.

at the positions $\pm \sqrt{h^{2}+2 t^{2}}$. The third (central) peak occurs at $\epsilon=0$, while the conductance reaches zero at $\pm h$. A particular situation occurs when $h_{1}=h_{2}=0$. The central peak, clearly seen in figure 3 for $h_{1}, h_{2} \neq 0$, does not occur then in the conductance. Since the system of three single-level QD's has three molecular-like states (denoted with the index 1, 2 and 3 for increasing energy), one could expect also three peaks in the conductance. However, the matrix elements of coupling between the molecular state $|\tilde{2}\rangle$ and the left and right leads vanish, the molecular state $|\tilde{2}\rangle$ becomes decoupled from the leads for $h_{1}=h_{2}=0$, which leads to the absence of the central peak in the conductance. As a consequence, one observes only two peaks in the linear conductance, situated at the positions $\epsilon= \pm \sqrt{2} t$, and the transmission reaches zero at $\epsilon=0$ (eigenvalue of the state $|\tilde{2}\rangle$ ). To show this more formally, let us perform the transformation of the dot's operators

$$
\begin{gathered}
\tilde{d}_{1 \sigma}=\frac{1}{2}\left(d_{1 \sigma}+\sqrt{2} d_{2 \sigma}+d_{3 \sigma}\right), \\
\tilde{d}_{2 \sigma}=\frac{1}{\sqrt{2}}\left(d_{1 \sigma}-d_{3 \sigma}\right)
\end{gathered}
$$




$$
\tilde{d}_{3 \sigma}=\frac{1}{2}\left(d_{1 \sigma}-\sqrt{2} d_{2 \sigma}+d_{3 \sigma}\right) .
$$

Assuming $\epsilon_{i \sigma}=\epsilon_{0}$ for $i=1,2,3$, the Hamiltonian of the isolated triple dots becomes then diagonal and takes the form

$$
\tilde{H}_{Q D}=\left(\epsilon_{0}+\sqrt{2} t\right) \tilde{d}_{1 \sigma}^{\dagger} \tilde{d}_{1 \sigma}+\epsilon_{0} \tilde{d}_{2 \sigma}^{\dagger} \tilde{d}_{2 \sigma}+\left(\epsilon_{0}-\sqrt{2} t\right) \tilde{d}_{3 \sigma}^{\dagger} \tilde{d}_{3 \sigma} \cdot
$$

In turn, the tunnelling Hamiltonian acquires then the form

$$
\tilde{H}_{T}=\sum_{\mathbf{k} \alpha \sigma} \sum_{i=1,3}\left(\tilde{V}_{i \mathbf{k} \sigma}^{\alpha} c_{\mathbf{k} \alpha \sigma}^{\dagger} \tilde{d}_{i \sigma}+\text { h.c. }\right)
$$

where

$$
\begin{gathered}
\tilde{V}_{1 \mathbf{k} \sigma}^{\alpha}=\frac{\sqrt{2}}{2} V_{2 \mathbf{k} \sigma}^{\alpha}, \\
\tilde{V}_{3 \mathbf{k} \sigma}^{\alpha}=-\frac{\sqrt{2}}{2} V_{2 \mathbf{k} \sigma}^{\alpha},
\end{gathered}
$$

and

$$
\tilde{V}_{2 \mathbf{k} \sigma}^{\alpha}=0
$$

This clearly shows that the central molecular-like level becomes effectively decoupled from the leads.

Let us consider now the influence of Coulomb correlation in the dots (nonzero parameters $U_{i}$ for $i=$ $1,2,3)$ and nonzero temperature on the spectra described above. Figure 4(a) corresponds to figure 2(f), except the Coulomb parameters which in figure $4(\mathrm{a})$ are nonzero, $U_{1}=U_{2}=U_{3}=1$, while in figure $2(\mathrm{f})$ they vanish, $U_{1}=U_{2}=U_{3}=0$. Owing to the nonzero Coulomb parameters, the number of peaks in the conductance becomes doubled in comparison to that in the case with vanishing Coulomb interaction. The side peaks are broad as in figure 2(f), whereas all the other four peaks are very narrow. Figure 4(b) shows the conductance spectrum for the same parameters as in figure 4(a), except temperature which now is higher. The peak heights are now smaller and the peaks become broader, as one might expect. Figures 4(c-e) show the conductance for a system with the Coulomb parameter vanishing for one or two dots. When the Coulomb parameter of a particular dot vanishes, the number of peaks decreases by one. In all the cases, however, the side peaks are broad while the others are narrow. Figure 4(f) corresponds to figure 4(a), but calculated for larger Coulomb correlation parameters, $U_{1}=U_{2}=U_{3}=4$. The whole spectrum becomes clearly split into two parts; one is similar to that for vanishing Coulomb parameters, and the second is its Coulomb counterpart, with a mirror plane somewhere in between the two parts.

All the results presented above were calculated for the parallel configuration of the leads' magnetic moments. Qualitatively similar results have been obtained for the
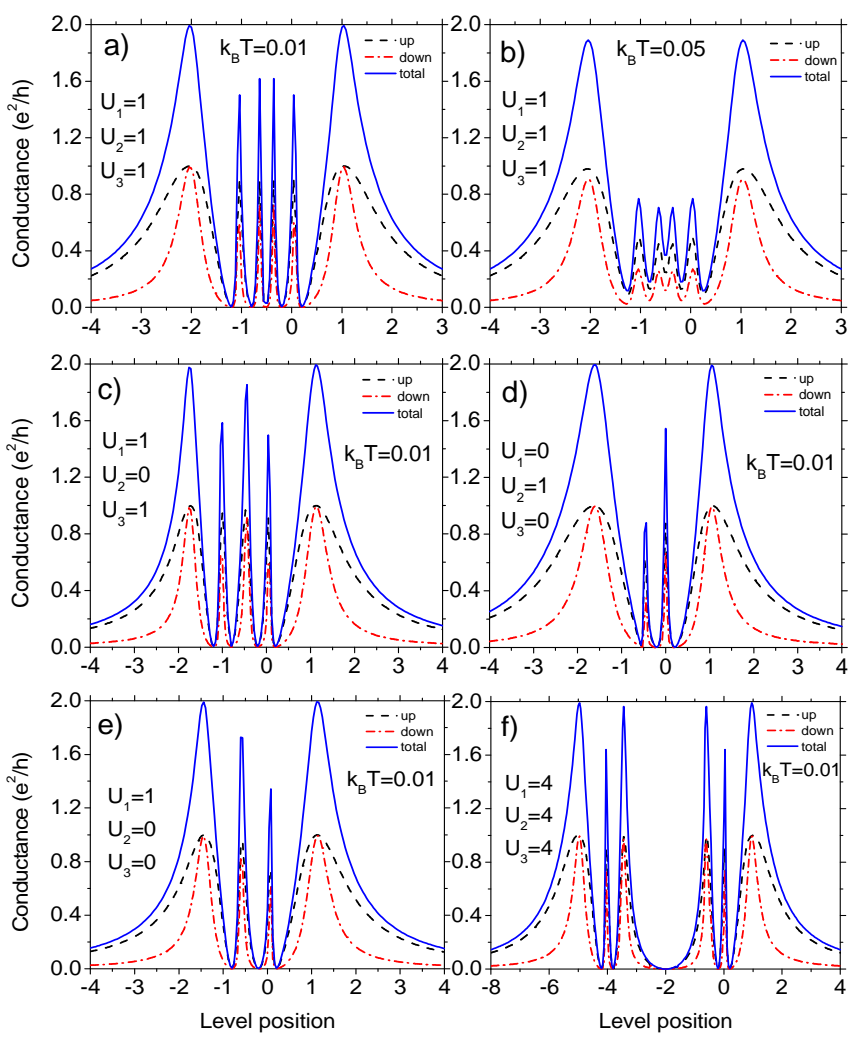

FIG. 4: Linear conductance in the parallel magnetic configuration, calculated as a function of the energy level $\epsilon_{2}$ of QD2 for indicated values of temperature and intra-dot Coulomb repulsion parameters $U_{1}, U_{2}, U_{3}$. The other parameters are: $t_{21}=t_{23}=0.8, h_{1}=h_{2}=0.2, p=0.4$, and $\gamma=1$. The energy is measured in the units of $\Gamma$.

antiparallel magnetic configuration. The quantitative difference leads to a nonzero TMR. In figure 5 we show TMR in the linear response regime as a function of the energy level $\epsilon_{2}$ of QD2. When the dots' levels are well above or well below the Fermi level of the leads, the TMR tends to the Julliere's value observed in planar magnetic tunnel junctions. When, in turn, the dot levels cross the Fermi energy, the situation becomes more complex and TMR significantly depends on the Coulomb parameters $U_{i}$. In the limit of $U_{i}=0$ for $i=1,2,3$, see figure $5(\mathrm{a})$, TMR shows then a clear peak structure and fluctuates about the Julliere's value. Qualitatively similar behavior of TMR was also found in transport through single quantum $\operatorname{dots}^{35}$. Variation of TMR with the level position is more complex for nonzero values of the Coulomb correlation parameters, as shown in figure 5(b). Significant resonance-like peaks in the TMR occur now at certain values of the level positions, at which the conductance (current) is strongly suppressed by the interference effects. Presence of such peaks in TMR is a consequence of the fact that the conditions for full destructive interference depend on magnetic configuration of the system for nonzero values of $U_{i}$. Outside the resonance-like peaks 

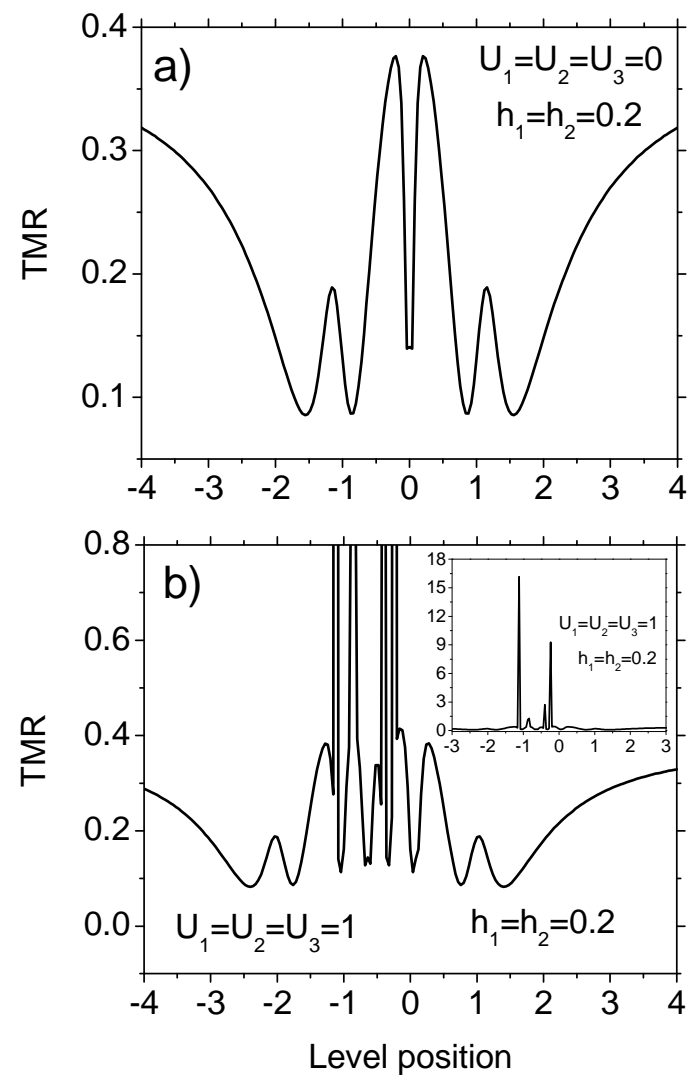

FIG. 5: TMR in the linear response regime, calculated as a function of the energy level $\epsilon_{2}$ of QD2 for indicated values of the intra-dot Coulomb repulsion parameters $U_{1}, U_{2}, U_{3}$, and for $t_{21}=t_{23}=0.8, h_{1}=h_{2}=0.2, p=0.4, \gamma=1$, and $k_{B} T=0.01$. The energy is measured in the units of $\Gamma$. the behavior of TMR is similar to that for vanishing $U_{i}$, except that the number of different peaks is larger due to the Coulomb counterparts in the conductance spectra.

\section{CONCLUSIONS}

In conclusion, we have calculated conductance of three coupled quantum dots. The conductance spectra, calculated in the Hartree-Fock approximation, clearly show presence of a central narrow peak, which resemblances the Dicke resonance in optical emission spectra of atoms. We have also shown, that the Coulomb correlations on the dots lead to doubling of the resonance peaks in the conductance. Additionally, we have calculated TMR effect associated with leads' magnetic moments rotation from antiparallel to parallel alignment, and shown that the intra-dot Coulomb correlation leads to some resonance-like enhancement of TMR at some values of the level positions.

\section{Acknowledgments}

This work, as part of the European Science Foundation EUROCORES Programme SPINTRA, was supported by funds from the Ministry of Science and Higher Education as a research project in years 2006-2009 and the EC Sixth Framework Programme, under Contract N. ERAS-CT2003-980409.
* Electronic address: piotrtroch@gmail.com

$\dagger$ Electronic address: barnas@amu.edu.pl

1 M. L. Guevara, F. Claro, P. A. Orellana, Phys. Rev. B 67, 195335 (2003).

${ }^{2}$ H. Lu, R. Lü, and B.-F. Zhu, J. Phys.: Condens. Matter 18, 8961 (2006).

${ }^{3}$ H. Lu, R. Lü, and B.-F. Zhu, Phys. Rev. B 71, 235320 (2005).

4 R. Lü, Z.-R. Liu, G.-M. Zhang, cond-mat/0504288.

${ }^{5}$ S. Y. Cho, R. H. McKenzie, K. Kang, C. K. Kim, J. Phys.: Condens. Matter 15, 1147 (2003).

${ }^{6}$ F. Chi, S.-S. Li, J. Appl. Phys. 99, 043705 (2006).

7 G.-H. Ding, C. K. Kim, K. Nahm, Phys. Rev. B 71, 205313 (2005).

8 A. C. Johnson, C.M. Marcus, M. P. Hanson, and A.C. Gossard, Phys. Rev. Lett. 93, 106803 (2004).

9 P. Trocha, J. Barnaś, phys. stat. sol. (b) 244, 2553 (2007).

10 D. Sztenkiel, R. Świrkowicz, J. Phys.: Condens. Matter 19, 176202 (2007).

11 D. Sztenkiel, R. Świrkowicz, J. Phys.: Condens. Matter 19, 386224 (2007).

12 P. Trocha, J. Barnaś, Phys. Rev. B 76, 165432 (2007).

13 Y. Tanaka and N. Kawakami, Phys. Rev. B 72, 085304
(2005).

14 C. Emary, cond-mat/0705.2934.

15 M. C. Rogge, R. J. Haug cond-mat/0707.2058v3

16 L. Gaudreau, A. S. Sachrajda, S. Studenikin, P. Zawadzki, A. Kam, J. Lapointe, AIP Conf. Proc. 893, 857 (2007).

17 T. Kuzmenko, K. Kikoin, Y. Avishai, Phys. Rev. B 69, 195109 (2004); Phys. Rev. Lett. 96, 046601 (2006).

18 D. S. Saraga, D. Loss, Phys. Rev. Lett. 90, 166803 (2003).

19 R. Žitko, J. Bonča, Phys. Rev. Lett. 98, 047203

20 M. L. Ladrón de Guevara, P. A. Orellana, Phys. Rev. B 73, 205303 (2006).

21 Z.-T. Jiang, Q.-F. Sun, Y. Wang, Phys. Rev. B 72, 045332 (2005).

22 I. V. Dinu, M. Tolea, A. Aldea, Phys. Rev. B 76, 113302 (2007).

23 I. Gómez, F. Dominguez-Adame, P. A. Orellana, J. Phys.: Condens. Matter 161613 (2004).

24 W.-Z. Wang, Phys. Rev. B 76, 115114 (2007).

25 T. Vorrath and T. Brandes, Phys. Rev. B 68, 035309 (2003).

26 T. Brandes and B. Kramer, Phys. Rev. Lett. 83, 3021 (1999).

27 T. Brandes, Phys. Rep. 408, 315 (2005). 
28 P.A. Orellana, F. Dominguez-Adame, E. Diez, Physica E 35,126 (2006).

29 P. A. Orellana, M. L. Ladrón de Guevara and F. Claro F Phys. Rev. B 70, 233315 (2004); P. A. Orellana, G. A. Lara, and E. V. Anda, Phys. Rev. B 74, 193315 (2006).

${ }^{30}$ R. H. Dicke, Phys. Rev. 89, 472 (1953).

31 R. H. Dicke, Phys. Rev. 93, 99 (1954).

32 R. G. DeVoe and R. G. Brewer, Phys. Rev. Lett. 76, 2049
(1996).

33 Y. Meir, N. S. Wingreen, Phys. Rev. Lett. 68 (1992) 2512.

34 A. P. Jauho, N. S. Wingreen, Y. Meir, Phys. Rev. B 50, 5528 (1994)

35 I. Weymann, J. Köenig, J. Martinek, J. Barnaś, and G. Schön, Phys. Rev. B 72, 115334 (2005). 